\title{
Retrospective follow-up analysis of the transcriptomic patterns of cytokines, cytokine receptors and chemokines at preconception and during pregnancy, in women with post-partum depression
}

\author{
MARIA CRISTINA PETRALIA ${ }^{1}$, EMANUELA MAZZON ${ }^{1}$, PAOLO FAGONE ${ }^{2}$, LUCA FALZONE ${ }^{2}$, \\ PLACIDO BRAMANTI $^{1}$, FERDINANDO NICOLETTI ${ }^{2}$ and MARIA SOFIA BASILE ${ }^{2}$ \\ ${ }^{1}$ IRCCS Centro Neurolesi ‘Bonino-Pulejo’, I-98124 Messina; \\ ${ }^{2}$ Department of Biomedical and Biotechnological Sciences, University of Catania, I-95123 Catania, Italy
}

Received June 3, 2019; Accepted July 8, 2019

DOI: $10.3892 /$ etm.2019.7774

\begin{abstract}
Post-partum depression (PPD) occurs in approximately $20 \%$ of women usually early following child delivery. PPD represents an important unmet medical need as it is frequently underdiagnosed and, as the neurobiology of the disease is limitedly known, no pathogenic-tailored approach is available and only symptomatic medications are used. In the present study, we carried out a DNA microarray analysis to evaluate the fluctuation of cytokines, cytokine receptors and chemokines during the preconception period, the 1st and 3 th trimester of pregnancy and the early post-partum period. The data demonstrated that, as compared to always-depressed patients and euthymic controls, women who developed PPD exhibited significant fluctuations in the levels of different cytokines and cytokine receptors, primarily related to tumor necrosis factor (TNF) and interleukin (IL)18. These data emphasize the role of the immune system in PPD. However, additional studies aimed at evaluating if and how these functional modifications of the immune system during pregnancy are related to the development of PPD warranted to confirm our findings.
\end{abstract}

\section{Introduction}

Post-partum depression (PPD) occurs in approximately $20 \%$ of women usually early following child delivery. Various risk factors for PPD have been identified, including obstetric complications, such as preeclampsia, and psychosocial factors, such as stressors during pregnancy, a low socioeconomic status, and a poor support status. PPD is predicted by a lifetime

Correspondence to: Professor Ferdinando Nicoletti, Department of Biomedical and Biotechnological Sciences, University of Catania, Via Santa Sofia 89, I-95123 Catania, Italy

E-mail: ferdinic@unict.it

Key words: post-partum depression, immune system, cytokines, chemokines history of major depression, PPD, bipolar disorder, premenstrual syndrome (PMS) and prenatal depressive symptoms at the end of term pregnancy (1).

PPD represents an important unmet medical need as it is frequently underdiagnosed and, as the neurobiology of the disease is limitedly known, no pathogenic-tailored approach is currently available and only symptomatic medications are used. Efforts are therefore warranted to understand the pathophysiology and the molecular basis of PPD, that may allow for the identification of successful treatment strategies.

Along this line of research, much attention has been paid to the possibility that dysregulated immune-inflammatory events may play a role in PPD. In particular, much interest has been placed on the role of hormones of the immune system, named cytokines, to the pathogenesis of PPD (2-4).

Based on preclinical and clinical studies, cytokines have been divided in at least 5 subfamilies: The pro-inflammatory Th1/Th17 cytokines, the anti-inflammatory Th2/Th3 cytokine and the Th9 cytokine, represented by IL9.

It has been shown that Th1 and Th17 cytokines primarily exert pro-inflammatory effects. They are produced by M1 macrophages, Th1 and Th17 cells and are comprised, among others, of IL1, TNF, IFN- $\gamma$, IL12, IL18, IL22 IL23, IL17. These cytokines are thought to contribute to the initiation of cell-mediated autoimmune diseases such as rheumatoid arthritis, type 1 diabetes, rheumatoid arthritis, multiple sclerosis and Guillain-Barre syndrome $(2,3,5)$.

On the other hand, anti-inflammatory cytokines (IL4, IL10, IL13, IL35, TGF- $\beta$ ) are primarily produced by M2 macrophages and Th2 and Th3 cells, and they are known to decrease inflammation and are implicated in IgE-mediated allergic diseases and eosinophil-mediated pathologies (6-9). Sometimes, such as in the case of systemic lupus erythematosus, it seems that the combined action of Th1/Th2 cytokines may be simultaneously involved in the pathogenesis of the disease (10). The precise role of IL9 in regulation of the immune responses is less defined and is receiving increased attention (4).

It is of interest that the balance between $\mathrm{Th} 1 / \mathrm{Th} 2$ and Th3 has been considered to be involved in the regulation, maintenance and progression of pregnancy in both preclinical 
and clinical studies, pointing to a strong association between maternal Th2-type immunity and successful pregnancy on the one hand, and between Th1-type immune reactivity and pregnancy loss, on the other (11).

It has also been hypothesized that an upregulated production of pro-inflammatory cytokines at preconception, during pregnancy and in the perinatal period may not only be detrimental for the outcome of the gestation but it may also contribute to the development of PPD. However, several conflicting reports have been generated in this regard. For example, one study found a positive correlation between depressive scores and circulating levels of interleukin (IL)6 and tumor necrosis factor (TNF)- $\alpha$, at an average of 15 weeks of gestation (12).

Another study demonstrated that the levels of IL6, IL10 and TNF- $\alpha$ were not significantly altered from third trimester to 12 weeks post-partum, but revealed an association between the late pregnancy levels of IL6 and IL10, and PPD symptoms (13).

In another study conducted on week 24th of gestation, the IL6 and TNF- $\alpha$ concentrations were unrelated to prenatal symptoms. However, a significant correlation was observed between increased levels of the Th1-related cytokine, IL12, the Th2-related cytokine, IL13, and the Th-9 related cytokine, IL9, and prenatal depressive and overall anxiety symptom scores. In addition, the interferon (IFN)- $\gamma /$ IL4 ratio and the concentration of the Th2-related cytokine, IL5, also correlated with depressive symptoms. Pregnancy-related anxiety score correlated positively with IL12, IL13, and anti-inflammatory IL10 concentrations (14).

Finally, another study conducted during the 3 th trimester of pregnancy in women with severe anxiety (SA) and severe depression (SD) reported that the highest levels of Th1- (IL6, TNF- $\alpha$, IL2, IFN- $\gamma$ ), Th17- (IL17A, IL22) and Th2-/Th9 (IL9, IL10, and IL13) related cytokines (reviewed in ref. 15) were observed in women who suffered from SD + SA. The women in the SA group exhibited higher concentrations of the Th1(IL6, TNF- $\alpha$, IL2, IFN- $\gamma$ ) and Th2- (IL4 and IL10) related cytokines compared with the controls. In addition, positive correlations were found between the Hamilton Depression Rating Scale (HDRS) scores and the Hamilton Anxiety Rating Scale (HARS) scores, and the levels of IL2, IL6, and TNF- $\alpha$ in the SA group, and between HDRS and Th1- (IL2, IL6, TNF- $\alpha$ ), Th2- (IL9, IL10, IL13) and Th17- (IL17A) cytokines in the SD + SA group. Following the implementation of the correlation analysis by gestational weeks, the correlations that remained significant were the following: HDRS and IL2, IL6, IL9 and IL17A in the SD + SA group. The HARS scores correlated with IL17A levels in the SA group, and with the IL17A, IL17F and IL2 levels in the SD + SA group (16).

These multiple outlined discrepancies may explained by several factors, including the different ethnicities of the women examined and underdiagnosed minor comorbidities that accompany the pregnancy, as well as the different gestational periods considered. Nonetheless, the data as a whole seem to support the concept that a dysregulated function of the immune system may occur during pregnancy, that is mirrored by an unbiased increase in the levels of Th1-, Th2and Th9-related cytokines, and may be associated with and/or predict PPD.
Along this line of research, in the present study, we carried out a DNA microarray study to evaluate the fluctuation of cytokines, cytokine receptors and chemokines during the preconception period, the 1st and 3th trimester of pregnancy and the early post-partum period. The data demonstrated that, as compared to the always-depressed patients and euthymic controls, women who developed PPD exhibited significant fluctuations in the levels of different cytokines and cytokine receptors primarily related to TNF and related ligands and receptors genes of the TNF superfamily and IL18.

These data are important as they emphasize the role of the immune system in PPD. However, additional studies are warranted to confirm our findings and to determine whether these functional modifications of the immune system during pregnancy may represent a therapeutic target for tailored therapeutic approaches for the prevention and treatment of PPD. It would also be important to establish whether abnormal patterns of circulating levels of cytokines and chemokines may offer a suitable biomarker for disease development and/or therapeutic response.

\section{Materials and methods}

Microarray dataset selection and clinical data of samples. For the evaluation of the expression levels of cytokines, chemokines and relative receptors in patients suffering from PPD, the publicly-available GSE45603 dataset was used. The dataset included whole-genome expression data of peripheral blood collected from women with no depressive symptoms (euthymic group, $n=27$ ), patients with PPD (PPD group, $n=17$ ) and patients who were continually depressed (always-depressed patients, $\mathrm{n}=18$ ). The present study is a third-party re-analysis of the GSE45603 dataset, that has already been used previously (17).

As indicated in (17), the patients matched for ethnicity, maternal age, diagnosis of DSM (namely, Diagnostic and Statistical Manual of Mental Disorders)-IV Axis I diseases (18), anxiety disorders, history of PPD, parity, pregnancy complications, thyroid-stimulating hormone levels, gestational age at delivery and delivery method. The Beck Depression Inventory (19), the 17-item Hamilton Rating Scale for Depression (20), and the Edinburgh Postnatal Depression Scale (EPDS) (21) and lifetime psychiatric diagnoses were used for the evaluation of depressive symptoms, using the Structured Clinical Interview for DSM-IV Axis I Disorders. In addition, no significant differences were found among the groups as regards the types or duration of prenatal exposure to psychotropic medications (17).

Peripheral blood was obtained at preconception, in the first and third trimester of pregnancy and at early post-partum, i.e., within the first 7 weeks after delivery (not all time points are available for all the patients).

Statistical analysis. The Illumina HT-12 v. 4.0 platform was used to generate whole-genome expression data. The raw data were transformed and normalized using the variance stabilizing normalization method and probes with detection P-value of $<0.01$ in at least $10 \%$ of the samples were used for analysis. Data are presented as the means \pm SD. A moderate permutation-based two-tailed Welch t-test was applied to 
estimate differences between groups. A Bonferroni-adjusted P-value $<0.05$ was considered to indicate a statistically significant difference. Analyses were performed using MultiExperiment Viewer Software 4.9.0.

\section{Results}

In this study, we aimed at evaluating the modulation of chemokines/cytokines and relative receptors in the whole blood of patients suffering from PPD, as compared to control patients, i.e., euthymic pregnant women who did not develop PPD and thereafter referred as euthymic women, and always-depressed patients. For this purpose, we interrogated the GSE45603 microarray dataset. Overall, the expression levels of 94 genes were investigated (Fig. 1).

Among the selected genes, a significant modulation of C-C motif chemokine receptor 2 (CCR2), interleukin 11 receptor subunit alpha (IL11RA), interleukin (IL)18 and tumor necrosis factor (TNF) superfamily member 14 (TNFSF14) expression was observed at preconception in women that will develop PPD as compared to euthymic women (Figs. 2 and S1). The IL11RA, IL18 and TNFSF14 expression levels were significantly upregulated, while that of CCR2 was downregulated. Notably, the CCR2 levels in the always-depressed patients seemed to exhibit a comparable trend to the PPD patients (although statistical significance was not reached), while the levels of IL11RA, IL18 and TNFSF14 in the always-depressed patients were similar to those of the euthymic patients (Figs. 2 and S1).

In the first trimester of pregnancy, the levels of 7 genes were found to be significantly altered in the group of women who will develop PPD, as compared to those without PPD (Figs. 3 and S2). In particular, the levels of IL10RB, IL1B, TNF, TNFRSF10B and TNFSF4, were upregulated, while a moderate, yet significant decrease in IL17RD and TNFSF15 levels was observed (Figs. 3 and S2).

In the third trimester of pregnancy, a significant upregulation in the levels of 6 genes was observed in the patients with PPD, in comparison to the control euthymic women and the always-depressed patients: CCL5, IL18RAP, TNFRSF17, TNFSF1B, TNF and TNFRSF10B (Figs. 4 and S3). The levels of none of these genes differed significantly between the euthymic women and always-depressed patients.

Among the genes encoding for cytokines, chemokines, and relative receptors that could be evaluated in the GSE45603 dataset, none of them were significantly modulated in the early post-partum period (within the first 7 weeks after delivery).

\section{Discussion}

The aim of this study was to evaluate transcriptomic differences in genes encoding for cytokines, chemokines and receptors, in a period of time ranging from preconception to the 3th trimester of pregnancy and early after the delivery period and to correlate the changes in peripheral transcriptomic profile to the development of PPD. The control group consisted of pregnant women that had not developed PPD (euthymic) or pregnant always-depressed patients. Overall, the expression levels of 94 genes were investigated by analyzing the publicly available GSE45603 microarray dataset. The use of whole-genome expression data has been largely used by our

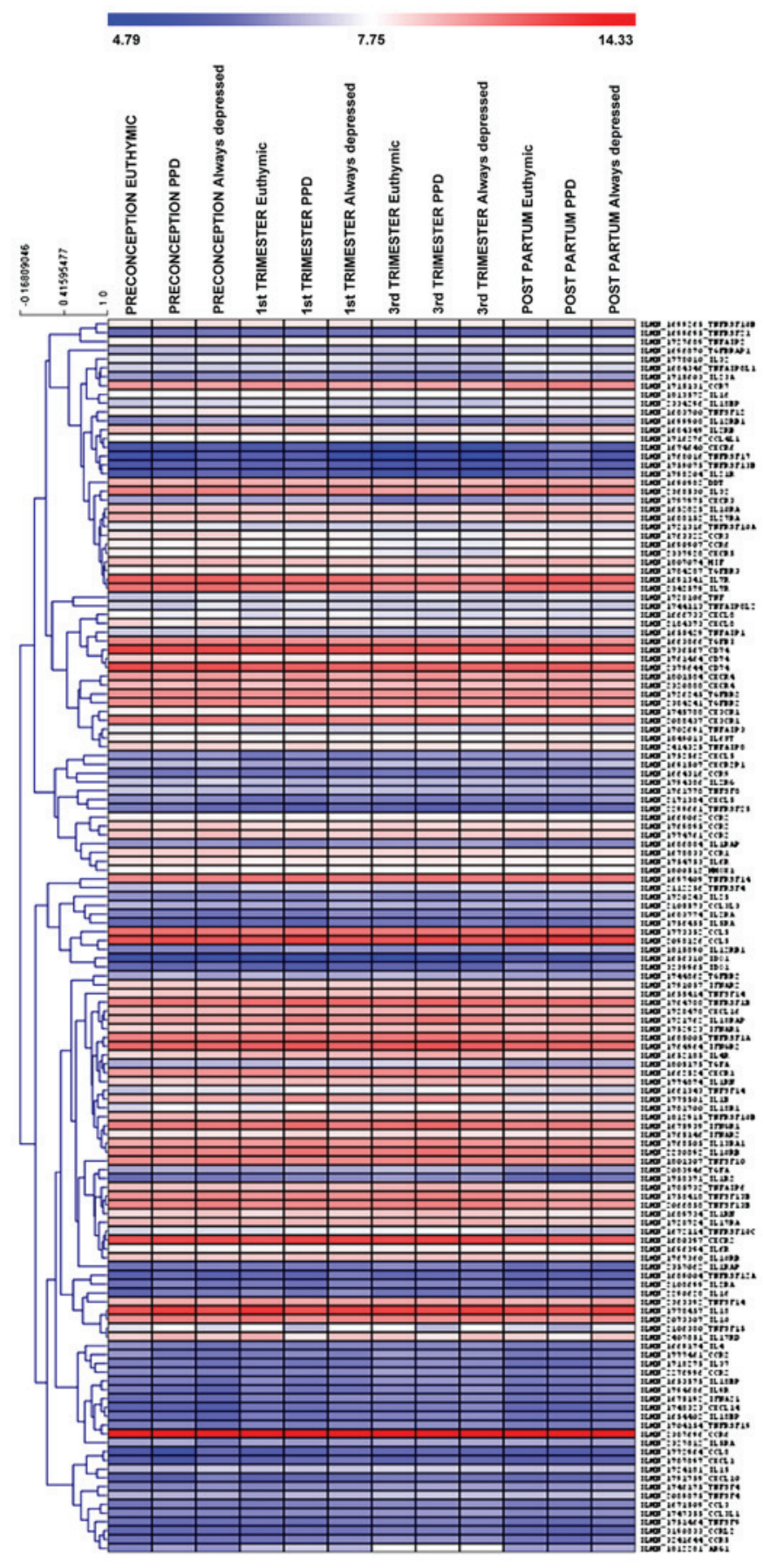

Figure 1. Heatmap showing the mean expression levels of the 94 cytokines, chemokines and corresponding receptors in the peripheral blood of euthymic patients, patients with PPD and always-depressed patients at preconception, 1st and 3rd trimester of pregnancy and in the early post-partum period, as evaluated in the GSE45603 dataset. PPD, post-partum depression.

group and other groups (22), to find pathogenic pathways and novel therapeutic targets for several disorders, including autoimmune diseases (23-28), cancer (29-32), degenerative (33) and infectious diseases (34).

The analysis of the data demonstrated that multiple modifications of the immune system, occurring at preconception and throughout the pregnancy period may be associated with the development of PPD.

It is of interest, from the pathogenic and possibly also the therapeutic point of view, to highlight that a modulation of the 

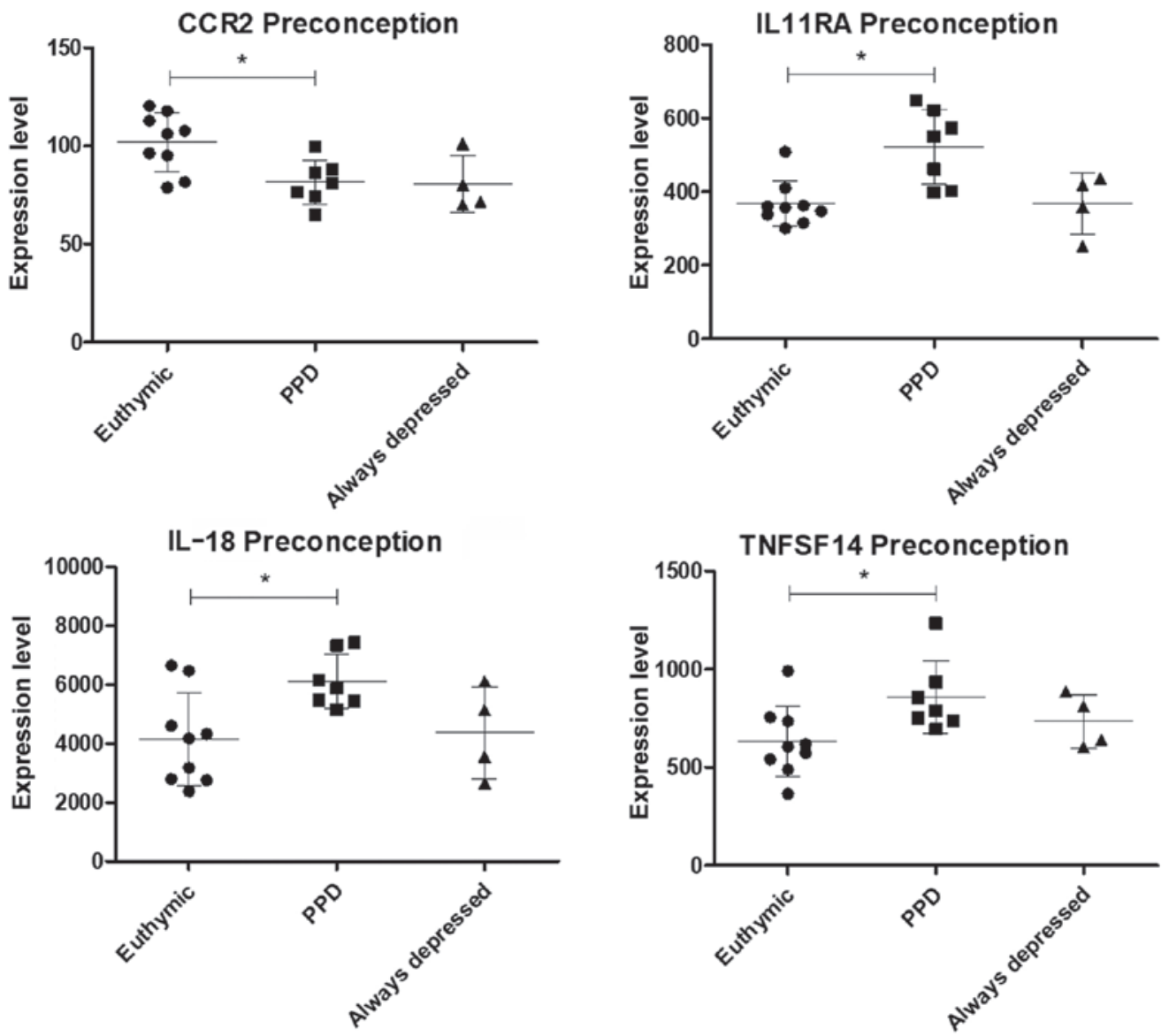

Figure 2. Scatterplots showing the expression levels of genes with significant differences in expression at preconception between the euthymic and patients with PPD, as evaluated in the GSE45603 dataset. PPD, post-partum depression.
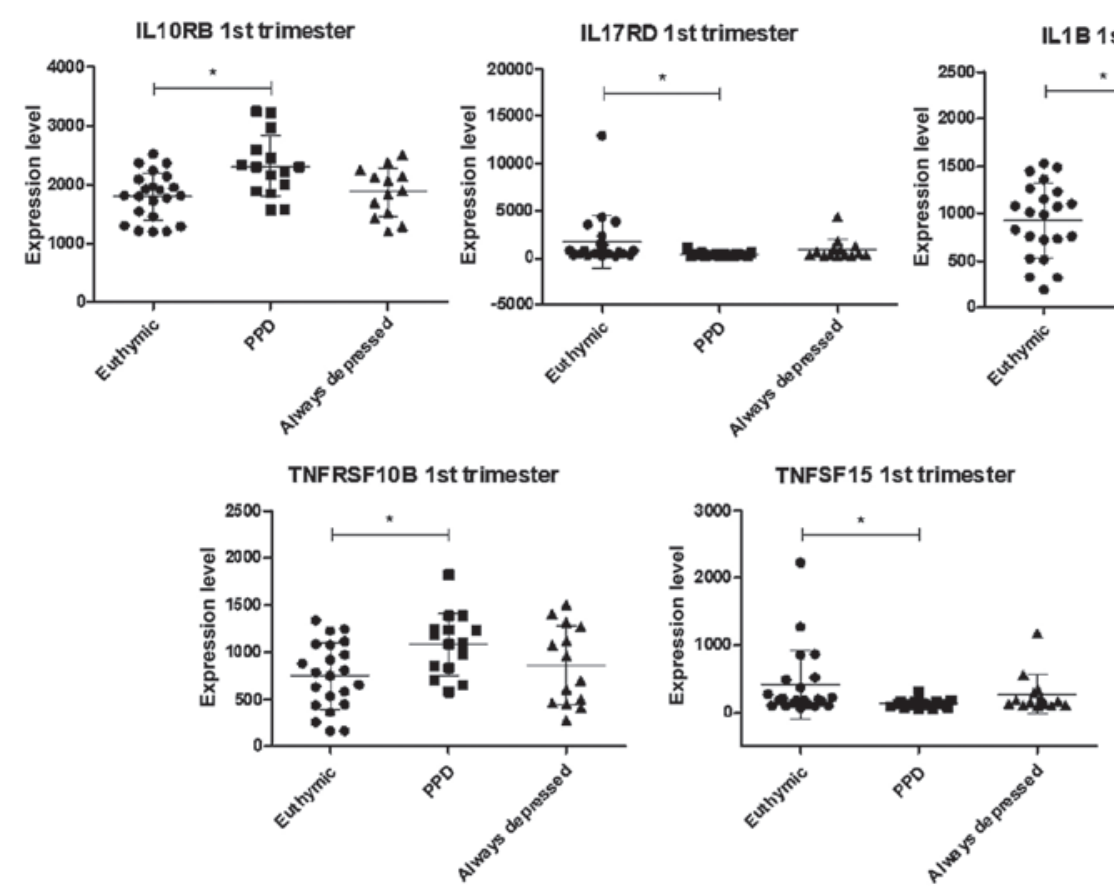

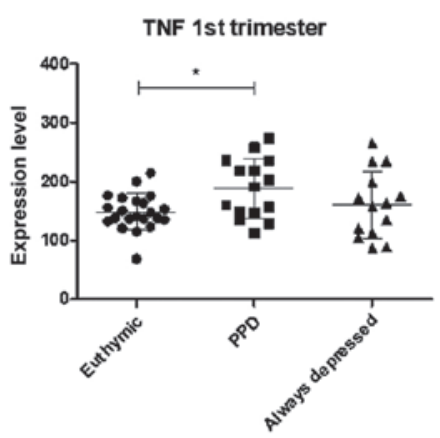

TNFSF4 1st trimester

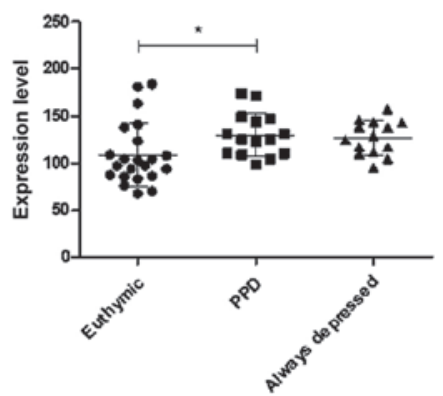

Figure 3. Scatterplots showing the expression levels of genes with significantly differences in expression at the 1st trimester of pregnancy between the euthymic and patients with PPD, as evaluated in the GSE45603 dataset. PPD, post-partum depression.

peripheral immune transcriptomic profile was observed in women already at the preconception period. In particular, at this time-point, with the exception of CCR2 expression, which was significantly downregulated, the levels of IL11RA, IL18 
CCL5 3rd trimester

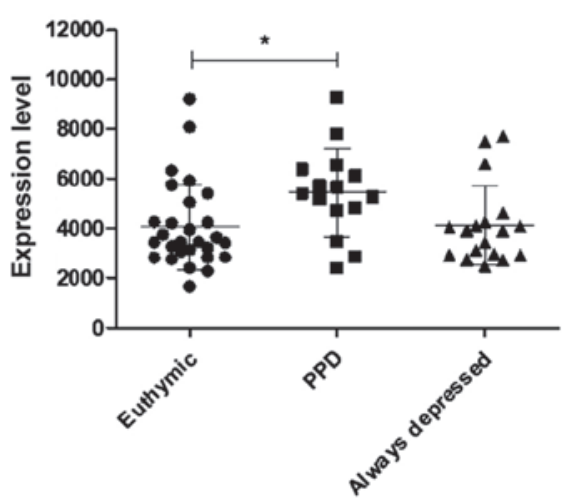

TNFSF1B 3rd trimester

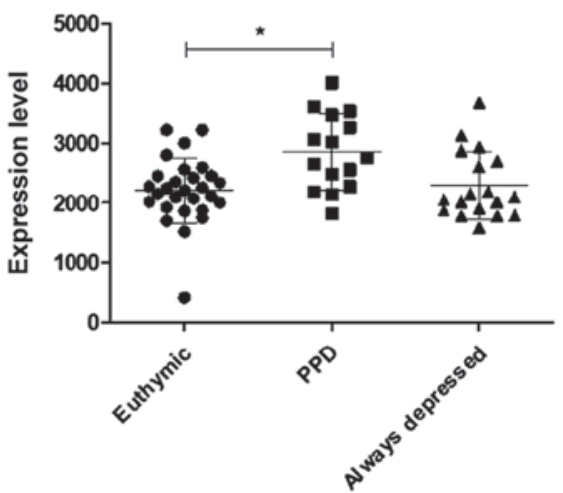

IL18RAP 3rd trimester

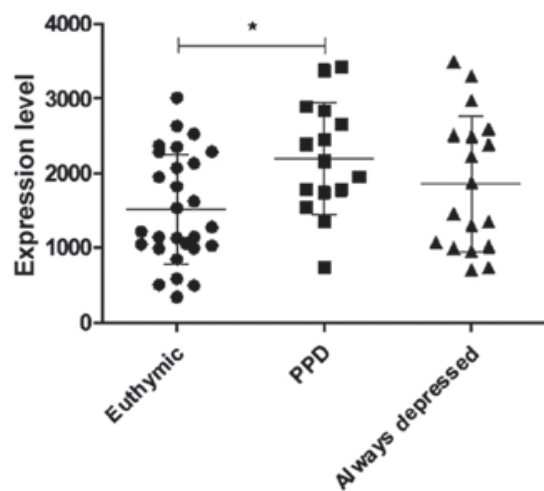

TNF 3rd trimester

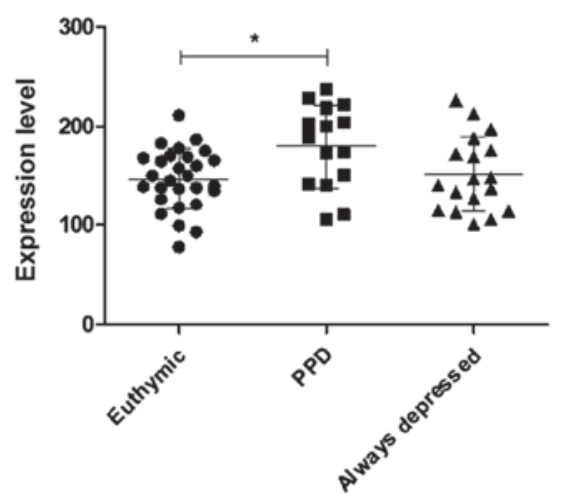

TNFRSF17 3rd trimester

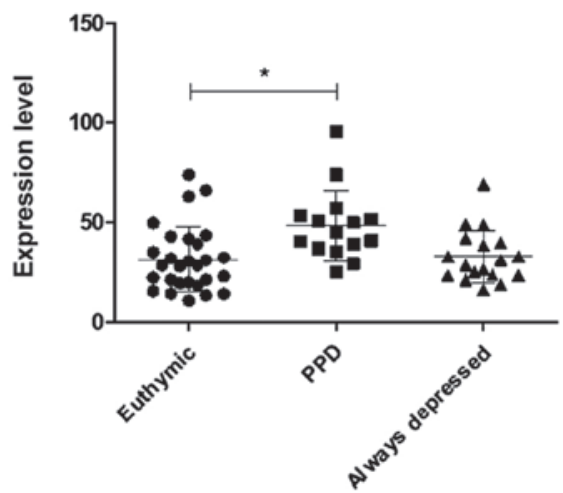

TNFRSF10B 3rd trimester

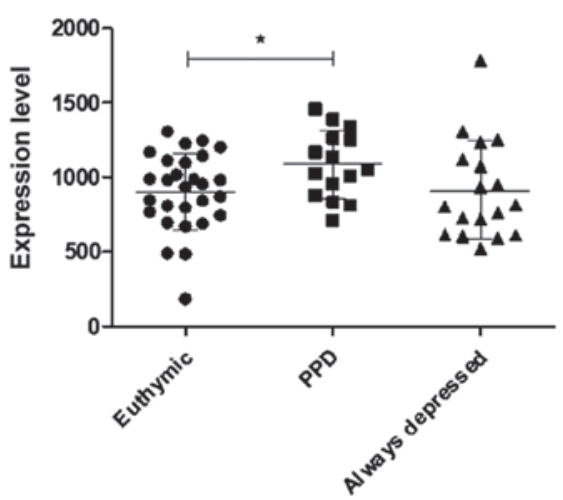

Figure 4. Scatterplots showing the expression levels of genes with significantly differences in expression at the 3rd trimester of pregnancy between the euthymic and patients with PPD, as evaluated in the GSE45603 dataset. PPD, post-partum depression.

and TNFSF14 were significantly upregulated as compared to the euthymic controls. Notably, the CCR2 levels in the always-depressed patients exhibited a comparable trend to those of the PPD patients (although the statistical significance was not reached), while the levels of IL11RA, IL18 and TNFSF14 in the always-depressed pregnant patients were similar to those of euthymic pregnant women.

During the first trimester of pregnancy, the levels of IL10RB, IL1B, TNF, TNFRSF10B and TNFSF4 were upregulated in women with PPD, as compared to euthymic pregnant women and always-depressed pregnant patients, along with a moderate, yet significant decrease in the levels of IL17RD and TNFSF15.

In the third trimester of pregnancy, the levels of CCL5, IL18RAP, TNFRSF17, TNFSF1B, TNF and TNFRSF10B were found to be significantly upregulated in women with PPD as compared to euthymic pregnant women and always-depressed patients.

Taken together, these observations are consistent with the hypothesis that an altered immunological profile, occurring already at preconception may predispose to deregulated immune responses during pregnancy that, in turn, may ultimately contribute to the development of PPD. The exact mode of action through which the pregnancy may further deregulate the functionality of the immune system during pregnancy remains to be determined. However, it is likely that hormonal modifications may eventually amplify preexisting abnormalities. This seems to be consistent with the observation that the transcriptomic abnormalities reverted in the early post-partum phase.

It also remains to be established whether and at what extent the immune abnormalities observed may contribute to the development of PPD. It has been postulated that a predominance of pro-inflammatory Th1/Th17, over anti-inflammatory Th2/Th3 cytokines, may contribute to the pathogenesis of major depressive disorders (MDD). A certain degree of variability in the fluctuation of mRNA transcripts among the different genes considered makes it difficult to firmly apply the cytokine hypothesis of MDD also to PPD. Nonetheless, it is interesting that, as regards the cytokines and cytokine receptors studied, an upregulation in the levels of Th1/Th17 cytokines over the Th2/Th3 counterparts seems to occur during the frame-time considered from preconception to the third trimester of pregnancy. In particular, the mRNA transcripts of the prototypical type 1 cytokine, IL18, were increased at preconception in women that will develop PPD. In these women, during the first trimester of pregnancy, this increase was followed by an increase in the mRNA transcripts of the pro-inflammatory cytokines, IL1B and TNF, along with a reduction in the levels of the receptors, IL10RB and IL17RD. The net consequence of these changes would result in upregulated Th1 and Th17 events as compared to Th2/Th3-mediated anti-inflammatory events that are further dampened by the reduction in the levels of the IL10 receptor $\mathrm{RB}$. In the third trimester of pregnancy, a similar scenario of pro- vs. anti-inflammatory cytokine-mediated events was 
observed, as there was a significant increase in the mRNA transcripts of TNF and IL18RAP. In particular, IL18RAP is needed for the assembly of IL18 to the IL18 receptor and activation of the subsequent signaling cascade (35-37) and its upregulated expression may indicate augmented signaling through the IL18/IL18 receptor system. As stated before, it is of interest that increased mRNA transcripts for IL18 were observed in women already at preconception, thus indicating that an upregulated profile of the IL18/IL18 receptor system occurs in women that will develop PPD.

The detailed mechanism through which, from the mechanistic and biomolecular point of view, upregulated Th1/Th17 events may induce PPD, remain to be determined. It has been proposed that pro-inflammatory cytokines, such as IL1ß, TNF- $\alpha$ and IFN- $\gamma$ may play multiple roles in the pathogenesis of MDD by activating monoamine reuptake, stimulating the hypothalamic-pituitary-adrenocortical (HPA) axis, upregulating oxidative events and decreasing the production of serotonin due to the increased activity of indolamine-2,3-dioxygenase (IDO) $(38,39)$.

A recent support of the possible role of pro-inflammatory cytokines to the pathogenesis of PPD via augmented tryptophan (TRP) synthesis comes from a recent study from Teshigawara et al who evaluated alterations in tryptophan metabolism during the pregnancy and post-partum periods. By using the EPDS, participants were divided into a non-depressive (ND) group, a post-partum depressive (PD) group, a temporary gestational depressive (TG) group, and a continuous depressive (CD) group. Blood samples were collected before and 1 month following delivery. The authors observed significant differences in the plasma levels of TRP metabolites during the pregnancy and post-partum periods between the women in the ND group and PD group, but not between those in the TG or CD group. In the PD group, the plasma levels of kynurenine (KYN) and kynurenic acid (KA), and KYN/TRP and $\mathrm{KA} / \mathrm{KYN}$ ratio during the pregnancy period were higher and the levels of 3-hydroxyanthranilic acid (3HAA) during the post-partum period was lower than those in the women in the ND group (40).

However, critical appraisal of these hypotheses is required, as cytokine elevation is not specific to depression. As stated above, PPD seems to represent a multifactorial syndrome and it is likely that the abnormal immune parameters may represent a cofactor able to increase the likelihood of its development.

That these immune abnormalities may not be the key mediator of PPD is also consistent with the observation that the symptoms of depression do not occur already during pregnancy, when many immune abnormalities occur. It is likely that the combined action of hormonal modifications observed during pregnancy and immune abnormalities may dictate the timeline of PPD occurrence. On the basis of these data, we favor the possibility that immune abnormalities, that polarize immune responses toward a Th1/Th17 mediated cytokine profile, may predispose pregnant women to developing PPD in the future.

It is worth noting that our transcriptomic analysis failed to demonstrate major abnormalities of genes for the several cytokines studied in the always-depressed patients. This is at a variance with several studies, including a recent meta-analysis that indicated multiple abnormalities of circulating cytokines in patients with MDD, such as increased levels of IL6, TNF- $\alpha$, IL10, the soluble IL2 receptor, CCL2, IL13, IL18, IL12, the IL1 receptor antagonist, and the soluble TNF receptor 2 and lower levels of interferon- $\gamma$ (41).

The reason for these discrepancies remains to be determined. However, it should be noted that the cytokines reported to be augmented in the meta-analysis were measured in the periphery blood and thus, in most cases, they also reflect production from non-immune cells, such as endothelial cells; thus, their circulating values do not necessarily reflect the specific production from mononuclear cells, as we have analyzed in this DNA microarray study. In addition, different ethnicities of patients with PPD and different medications and comorbidity may contribute to the varying results obtained.

Another point of view that emerges from the analysis of this study is the abnormal expression of TNFSF14, that was increased at preconception in the women with PPD. In addition, the levels of TNFRSF10B, TNFSF15 and TNFSF4 were found to be altered at the 1st trimester, and those of TNFRSF17, TNFSF1B and TNFRSF10B were also found to be altered at the 3rd trimester.

These genes belong to ligands and receptors of the TNF superfamily and are primarily, but not exclusively, involved in the control of apoptosis. These ligands and receptors are expressed on different cell types and are also implicated in several biological processes, including osteoclastogenesis and the regulation of immune-inflammatory responses (42).

To the best of our knowledge, this is the first study to observe a significant fluctuation in the mRNA transcripts of these molecules from preconception to pregnancy, in women developing PPD, and to suggest their putative role in the pathogenesis of the disease. However, further studies are required to confirm our findings and hypotheses.

In addition, in this case, profound differences in transcriptomic profiles with the always-depressed patients were observed. This underlines and enforces previously described immunopathogenic differences between PPD and MDD also outlined by the different therapeutic approaches thus far utilized and that are being studied $(43,44)$.

It is difficult to predict the eventual translational relevance of our present findings to the clinical setting. However, the present finding of upregulated Th1/Th17 balance in PPD may have both diagnostic and therapeutic relevance if it may allow early diagnosis of women at risk for PPD and early therapeutic or even prophylactic intervention.

To the best of our knowledge, this is the first study to demonstrate transcriptomic modifications of these genes in women that will develop PPD. However, the precise role of these genes in the contribution of the disease remains to be determined.

\section{Acknowledgements}

Not applicable.

\section{Funding}

The present study was supported by current research funds 2019 of IRCCS Centro Neurolesi 'Bonino-Pulejo', Messina-Italy. 


\section{Availability of data and materials}

The dataset analyzed in the current study is available from the Gene Expression Omnibus website (https://www.ncbi.nlm.nih. gov/geo/) with the Accession no. GSE45603.

\section{Authors' contributions}

MCP, FN and MSB made substantial contributions to the conception and design of the study. EM, PF, LF, PB and MCP analyzed the data and prepared the figures. MCP, MSB and FN interpreted the data and wrote the manuscript. All authors have read and approved the final version of the manuscript.

\section{Ethics approval and consent to participate}

The patient data were obtained from a publicly available dataset; thus, no ethics approval was required.

\section{Patient consent for publication}

Not applicable.

\section{Competing interests}

The authors declare that they have no competing interests.

\section{References}

1. Roomruangwong C, Anderson G, Berk M, Stoyanov D Carvalho AF and Maes M: A neuro-immune, neuro-oxidative and neuro-nitrosative model of prenatal and postpartum depression Prog Neuropsychopharmacol Biol Psychiatry 81: 262-274, 2018.

2. Roeleveld DM and Koenders MI: The role of the Th17 cytokines IL-17 and IL-22 in Rheumatoid Arthritis pathogenesis and developments in cytokine immunotherapy. Cytokine 74: 101-107, 2015.

3. Zhang H-L, Zheng X-Y and Zhu J: Th1/Th2/Th17/Treg cytokines in Guillain-Barré syndrome and experimental autoimmune neuritis. Cytokine Growth Factor Rev 24: 443-453, 2013.

4. Chakraborty S, Kubatzky KF and Mitra DK: An update on interleukin-9: From its cellular source and signal transduction to its role in immunopathogenesis. Int J Mol Sci 20: 2113, 2019.

5. Dujmovic I, Mangano K,Pekmezovic T, Quattrocchi C, Mesaros S, Stojsavljevic N, Nicoletti F and Drulovic J: The analysis of IL-1 beta and its naturally occurring inhibitors in multiple sclerosis: The elevation of IL-1 receptor antagonist and IL-1 receptor type II after steroid therapy. J Neuroimmunol 207: 101-106, 2009.

6. Ayakannu R, Abdullah NA, Radhakrishnan AK, Lechimi Raj V and Liam CK: Relationship between various cytokines implicated in asthma. Hum Immunol: May 1, 2019 (Epub ahead of print). doi: 10.1016/j.humimm.2019.04.018.

7. Nicoletti F, Mancuso G, Cusumano V, Di Marco R, Zaccone P, Bendtzen K and Teti G: Prevention of endotoxin-induced lethality in neonatal mice by interleukin-13. Eur J Immunol 27: 1580-1583, 1997.

8. Conway TF, Hammer L, Furtado S, Mathiowitz E, Nicoletti F, Mangano K, Egilmez NK and Auci DL: Oral delivery of particulate transforming growth factor beta 1 and all-trans retinoic acid reduces gut inflammation in murine models of inflammatory bowel disease. J Crohn's Colitis 9: 647-658, 2015.

9. Nicoletti F, Di Marco R, Patti F, Reggio E, Nicoletti A, Zaccone P, Stivala F, Meroni PL and Reggio A: Blood levels of transforming growth factor-beta 1 (TGF-beta1) are elevated in both relapsing remitting and chronic progressive multiple sclerosis (MS) patients and are further augmented by treatment with interferon-beta $1 \mathrm{~b}$ (IFN-beta1b). Clin Exp Immunol 113: 96-99, 1998.

10. Barcellini W, Rizzardi GP, Borghi MO, Nicoletti F, Fain C, Del Papa N and Meroni PL: In vitro type- 1 and type- 2 cytokine production in systemic lupus erythematosus: Lack of relationship with clinical disease activity. Lupus 5: 139-145, 1996.
11. Raghupathy R: Pregnancy: Success and failure within the Th1/Th2/Th3 paradigm. Semin Immunol 13: 219-227, 2001.

12. Christian LM, Franco A, Glaser R and Iams JD: Depressive symptoms are associated with elevated serum proinflammatory cytokines among pregnant women. Brain Behav Immun 23: 750-754, 2009.

13. Simpson W, Steiner M, Coote M and Frey BN: Relationship between inflammatory biomarkers and depressive symptoms during late pregnancy and the early postpartum period: A longitudinal study. Br J Psychiatry 38: 190-196, 2016.

14. Karlsson L, Nousiainen N, Scheinin NM, Maksimow M, Salmi M, Lehto SM, Tolvanen M, Lukkarinen H and Karlsson H: Cytokine profile and maternal depression and anxiety symptoms in mid-pregnancy-the FinnBrain Birth Cohort Study. Arch Women Ment Health 20: 39-48, 2017.

15. Meylan F and Siegel RM: TNF superfamily cytokines in the promotion of Th9 differentiation and immunopathology. Semin Immunopathol 39: 21-28, 2017.

16. Leff Gelman P, Mancilla-Herrera I, Flores-Ramos M, Saravia Takashima MF, Cruz CoronelFM, Cruz Fuentes C,Pérez Molina A, Hernández-Ruiz J, Silva-Aguilera FS, Farfan-Labonne B, et al: The cytokine profile of women with severe anxiety and depression during pregnancy. BMC Psychiatry 19: 104, 2019.

17. Mehta D, Newport DJ, Frishman G, Kraus L, Rex-Haffner M, Ritchie JC, Lori A, Knight BT, Stagnaro E, Ruepp A, et al: Early predictive biomarkers for postpartum depression point to a role for estrogen receptor signaling. Psychol Med 44: 2309-2322, 2014.

18. Lobbestael J, Leurgans M and Arntz A: Inter-rater reliability of the structured clinical interview for DSM-IV axis I disorders (SCID I) and axis II disorders (SCID II). Clin Psychol Psychother 18: 75-79, 2011.

19. Richter P, Werner J,Heerlein A, Kraus A and Sauer H: On the validity of the Beck Depression Inventory. A review. Psychopathology 31: 160-168, 1998.

20. BoboWV,AngleróGC, Jenkins G,Hall-FlavinDK, WeinshilboumR and Biernacka JM: Validation of the 17-item Hamilton Depression Rating Scale definition of response for adults with major depressive disorder using equipercentile linking to Clinical Global Impression scale ratings: Analysis of Pharmacogenomic Research Network Antidepressant Medication Pharmacogenomic Study (PGRN-AMPS) data. Hum Psychopharmacol 31: 185-192, 2016.

21. Usuda K, Nishi D, Okazaki E, Makino M and Sano Y: Optimal cut-off score of the Edinburgh Postnatal Depression Scale for major depressive episode during pregnancy in Japan. Psychiatry Clin Neurosci 71: 836-842, 2017.

22. Gustafsson M, Edström M, Gawel D, Nestor CE, Wang H, Zhang H, Barrenäs F, Tojo J, Kockum I, Olsson T, et al: Integrated genomic and prospective clinical studies show the importance of modular pleiotropy for disease susceptibility, diagnosis and treatment. Genome Med 6: 17, 2014.

23. Fagone P, Mazzon E, Cavalli E, Bramanti A, Petralia MC, Mangano K, Al-Abed Y, Bramati P and Nicoletti F: Contribution of the macrophage migration inhibitory factor superfamily of cytokines in the pathogenesis of preclinical and human multiple sclerosis: In silico and in vivo evidences. J Neuroimmunol 322: 46-56, 2018.

24. Mangano K, Cavalli E, Mammana S, Basile MS, Caltabiano R, Pesce A, Puleo S, Atanasov AG, Magro G, Nicoletti F, et al: Involvement of the $\mathrm{Nrf} 2 / \mathrm{HO}-1 / \mathrm{CO}$ axis and therapeutic intervention with the CO-releasing molecule CORM-A1, in a murine model of autoimmune hepatitis. J Cell Physiol 233: 4156-4165, 2018.

25. Mammana S, Bramanti P, Mazzon E, Cavalli E, Basile MS, Fagone P, Petralia MC, McCubrey JA, Nicoletti F and Mangano K: Preclinical evaluation of the PI3K/Akt/mTOR pathway in animal models of multiple sclerosis. Oncotarget 9: 8263-8277, 2018.

26. Fagone P, Muthumani K, Mangano K, Magro G, Meroni PL, Kim JJ, Sardesai NY, Weiner DB and Nicoletti F: VGX-1027 modulates genes involved in lipopolysaccharide-induced Toll-like receptor 4 activation and in a murine model of systemic lupus erythematosus. Immunology 142: 594-602, 2014.

27. Fagone P, Mazzon E, Mammana S, Di Marco R, Spinasanta F, Basile MS, Petralia MC, Bramanti P, Nicoletti F and Mangano K: Identification of $\mathrm{CD} 4^{+} \mathrm{T}$ cell biomarkers for predicting the response of patients with relapsing remitting multiple sclerosis to natalizumab treatment. Mol Med Rep 20: 678-684, 2019.

28. Nicoletti F, Mazzon E, Fagone P, Mangano K, Mammana S, Cavalli E, Basile MS, Bramanti P, Scalabrino G, Lange A, et al: Prevention of clinical and histological signs of MOG-induced experimental allergic encephalomyelitis by prolonged treatment with recombinant human EGF. J Neuroimmunol 332: 224-232, 2019. 
29. Presti M, Mazzon E, Basile MS, Petralia MC, Bramanti A, Colletti G, Bramanti P, Nicoletti F and Fagone P: Overexpression of macrophage migration inhibitory factor and functionally-related genes, D-DT, CD74, CD44, CXCR2 and CXCR4, in glioblastoma. Oncol Lett 16: 2881-2886, 2018.

30. Fagone P, Caltabiano R, Russo A, Lupo G, Anfuso CD Basile MS, Longo A, Nicoletti F, De Pasquale R, Libra M, et al: Identification of novel chemotherapeutic strategies for metastatic uveal melanoma. Sci Rep 7: 44564, 2017.

31. Basile MS, Mazzon E, Russo A, Mammana S, Longo A, Bonfiglio V, Fallico M, Caltabiano R, Fagone P, Nicoletti F, et al: Differential modulation and prognostic values of immune-escape genes in uveal melanoma. PLoS One 14: e0210276, 2019.

32. Mangano K, Mazzon E, Basile MS, Di Marco R, Bramanti P, Mammana S, Petralia MC, Fagone P and Nicoletti F: Pathogenic role for macrophage migration inhibitory factor in glioblastoma and its targeting with specific inhibitors as novel tailored therapeutic approach. Oncotarget 9: 17951-17970, 2018.

33. Fagone P,ManganoK,MammanaS,Pesce A,Pesce A,CaltabianoR, Giorlandino A, Portale TR, Cavalli E, Lombardo GA, et al: Identification of novel targets for the diagnosis and treatment of liver fibrosis. Int J Mol Med 36: 747-752, 2015.

34. Fagone P,Nunnari G,Lazzara F, Longo A, Cambria D, Distefano G, Palumbo M, Nicoletti F, Malaguarnera L and Di Rosa M: Induction of OAS gene family in HIV monocyte infected patients with high and low viral load. Antiviral Res 131: 66-73, 2016.

35. Born TL, Thomassen E, Bird TA and Sims JE: Cloning of a novel receptor subunit, AcPL, required for interleukin-18 signaling. J Biol Chem 273: 29445-29450, 1998.

36. Torigoe K, Ushio S, Okura T, Kobayashi S, Taniai M, Kunikata T, Murakami T, Sanou O, Kojima H, Fujii M, et al: Purification and characterization of the human interleukin-18 receptor. J Biol Chem 272: 25737-25742, 1997.

37. Salcedo R, Worschech A, Cardone M, Jones Y, Gyulai Z, Dai RM Wang E, Ma W, Haines D, O'hUigin C, et al: MyD88-mediated signaling prevents development of adenocarcinomas of the colon: Role of interleukin 18. J Exp Med 207: 1625-1636, 2010.
38. Lichtblau N, Schmidt FM, Schumann R, Kirkby KC and Himmerich H: Cytokines as biomarkers in depressive disorder: Current standing and prospects. Int Rev Psychiatry 25: 592-603, 2013.

39. Quak J, Doornbos B, Roest AM, Duivis HE, Vogelzangs N, Nolen WA, Penninx BW, Kema IP and de Jonge P: Does tryptophan degradation along the kynurenine pathway mediate the association between pro-inflammatory immune activity and depressive symptoms? Psychoneuroendocrinology 45: 202-210, 2014.

40. Teshigawara T, Mouri A, Kubo H, Nakamura Y, Shiino T, Okada T, Morikawa M, Nabeshima T, Ozaki N, Yamamoto Y, et al: Changes in tryptophan metabolism during pregnancy and postpartum periods: Potential involvement in postpartum depressive symptoms. J Affect Disord 255: 168-176, 2019.

41. Köhler CA, Freitas TH, Maes M, de Andrade NQ, Liu CS, Fernandes BS, Stubbs B, Solmi M, Veronese N, Herrmann N, et al: Peripheral cytokine and chemokine alterations in depression: A meta-analysis of 82 studies. Acta Psychiatr Scand 135: 373-387, 2017.

42. Xu W, Xu Z, Huang L, Qin EQ, Zhang JL, Zhao P, Tu B, Shi L, Li WG and Chen WW: Transcriptome sequencing identifies novel immune response genes highly related to the severity of human adenovirus type 55 infection. Front Microbiol 10: 130, 2019.

43. Dennis C-LE and Stewart DE: Treatment of postpartum depression, part 1: A critical review of biological interventions. J Clin Psychiatry 65: 1242-1251, 2004.

44. Frieder A, Fersh M, Hainline R and Deligiannidis KM: Pharmacotherapy of postpartum depression: Current approaches and novel drug development. CNS Drugs 33: 265-282, 2019.

This work is licensed under a Creative Commons Attribution-NonCommercial-NoDerivatives 4.0 International (CC BY-NC-ND 4.0) License. 